Meta

Journal des traducteurs

Translators' Journal

\title{
Traduction et culture : quelques proverbes africains traduits
}

\section{Catherine Sumner-Paulin}

Volume 40, numéro 4, décembre 1995

URI : https://id.erudit.org/iderudit/003802ar

DOI : https://doi.org/10.7202/003802ar

Aller au sommaire du numéro

Éditeur(s)

Les Presses de l'Université de Montréal

ISSN

0026-0452 (imprimé)

1492-1421 (numérique)

Découvrir la revue

Citer cet article

Sumner-Paulin, C. (1995). Traduction et culture : quelques proverbes africains traduits. Meta, 40(4), 548-555. https://doi.org/10.7202/003802ar

\section{Résumé de l'article}

L'auteur s'intéresse aux problèmes des traducteurs qui travaillent avec des langues de cultures éloignées (par ex. l'Afrique traditionnelle et le monde de l'homme blanc). Elle examine la traduction de quelques proverbes africains et montre les obstacles linguistiques et sociolinguistiques rencontrés par les traducteurs. EIle propose une approche plus littérale de la traduction, ce qui permettrait de mieux respecter I'identité de communautés culturellement éloignées. 


\title{
TRADUCTION ET CULTURE : QUELQUES PROVERBES AFRICAINS TRADUITS
}

\author{
CATHERINE SUMNER-PAULIN
}

Université de Be'suncom, Be'sancom, Fiancer

\begin{abstract}
Résumé
L'auteur s'intéresse aur problemes des traducteurs qui travaillent aser des langues de

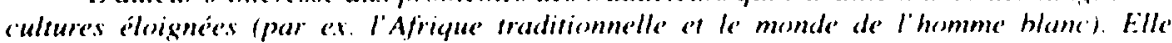

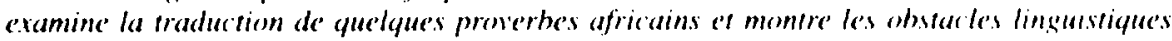

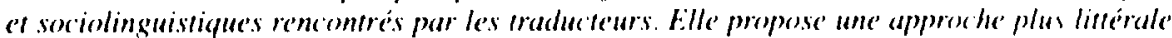

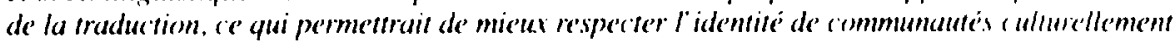
éloignées.
\end{abstract}

\section{Abstract}

The author discusses the problems faced by translators working with languages of

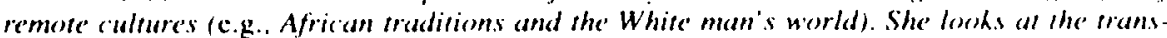
lation of a fen African proverbs and posints ont the linguistic and sociolinguistic diffic alties facing the translator. She recommends a rather literal translation approath so as to respect the identity of culturally remote communities

On sait que la traduction est souvent liée à un problème de transposition ou de modulation, dans le domaine grammatical et plus encore dans le domaine lexical. Au-delà des considérations linguistiques, la traduction fait apparaître les rapports étroits de l'être humain avec son entourage physique et métaphysique et. de ce fait, ne peut être dissociéc du contexte socioculturel. Sans remettre en cause la nécessité de la traduction, peut-on envisager qu'elle soit la transmission d'un sens identique à travers la différence des langues et des cultures?

Retracer très brièvement l'histoire de la traduction (Martin 1982:88-89) permet de voir à quel point elle est envisagée différemment en fonction de l'époque et des convictions du traducteur. Dans les sociétés primitives. la traduction était frappée d’interdits. Les premières traductions sont liées à la propagation des religions. Elle acquiert une nouvelle dimension grâce à l'essor du commerce aux XVI" el XVII" siècles. À cette époque, traduire devient véritablement une opération de réceriture : la "traduction-annexion" consiste à réécrire et à rendre conforme aux normes de la société de la langue cible le texte traduit. Les romantiques, pour qui le but à atteindre semblerait être d'accéder aux différences culturelles, développent la tendance inverse el ont une conception littéraliste de la traduction. L'interprétation du texte source est réduite au minimum, et traduire est une opération de calque. Industrialisation et clivage entre traduction poétique ou littéraire et traduction technique ou scientifique cö̈ncident. La première est réputéc difficile, voire irréalisable : la seconde se prête à des opérations de transcodage.

Fedorov (195.3 : 22) souligne que pour traduire, il faut certes connaître des langues. mais que la difficulté réside dans le fait que traduire de lat littérature est une opération littéraire : traduire des vers, une opération pótique : doubler un film. comme dans les autres cas, une opération sui gone'ris.

Si l'on admet que la traduction est une réinterprétation du texte source, puis une réécriture de celui-ci et, par conséquent, une activité créatrice, elle n'est pas réductible à un exercice linguistique. 
Dans le cas de traductions littéraires pour lesquelles il s'agit non seulement de passer d'une langue à une autre, mais d'établir un pont entre des cultures très différentes l'une de l'autre, les difficultés liées à la traduction sont, d'une part. d'ordre linguistique, et stylistique d'autre part, et même essentiellement d'ordre sociolinguistique. Toute traduction implique qu'un message existant dans une langue donnée soit transformé de manière à être compris par un lecteur qui ne connaît pas cette langue. Dans le cas qui vient d'être mentionné. le lecteur ne connaît pas non plus la culture dont il s'agit. Le traducteur littéraire ne peut jamais dire dans sa langue tout ce que l'auteur a exprimé dans la sienne. mais il peut le plus souvent dire ce que l'auteur aurait dit s'il s'était servi du langage du traducteur. Que se passe-t-il quand les réalités extra-linguistiques décrites par un autcur n'existent pas dans la langue du traducteur? Doit-on transposer d'un système de références à un autre au détriment du contenu socioculturel du texte source ? Préférerat-on calquer au maximum le texte cible sur le texte source afin que la traduction baigne dans la tradition linguistique et culturelle de la langue source, au risque de ne pas être aisément décodable?

Les obstacles linguistiques que rencontre tout traducteur sont tout d'abord d'ordre sémantique. Ainsi, une première classe de problèmes que pose la traduction relève de l'accessibilité ou non au sens du texte source. Ce sont des problèmes d'autant plus aigus lorsqu'il y a un grand décalage culturel entre le texte source et le lecteur potentiel du texte cible. Mounin écrit : «Pour traduire un texte écrit dans une langue étrangère. deux conditions sont nécessaires : connaître la langue et connaître la civilisation dont parle cette langue (la vie, la culture, l'ethnographie)" (Mounin 1976: 44). Par extension, pour comprendre un texte traduit, il est nécessaire d'avoir un minimum de connaissances des réalités extra-linguistiques auxquelles renvoie le texte source. Nous ne croyons pas à la possibilité théorique d'une coïncidence exacte entre deux termes d'un même champ lexical ou entre deux structures syntaxiques, dans deux langues différentes. Celle-ci s'amenuise encore en l'absence de fore "parenté culturelle" entre deux communautés linguistiques. Les échanges communicationnels peuvent poser des problèmes d'interprétation des intentions connotées de l'émetteur. Au niveau de la traduction. la connotation (comme les niveaux de langue) relève non seulement de la stylistique, mais aussi de considérations beaucoup plus vastes d'ordre sociolinguistique.

Selon Dubois (1973: 139), «la connotation est constituée par les éléments subjectifs ou variables selon les contextes de la signification d'une unité lexicale", par opposition à la dénotation qui en est l'élément stable, non subjectif et analysable hors du discours. Bien que les connotations d'un mot puissent varier d'un émetteur à un autre. elles sont tout de même partagées, dans une large mesure, par la communauté linguistique. D'après Chuquet et Paillard (1989:21), "certaines connotations échappent inévitablement à la traduction». Ils ajoutent plus loin que « exploitation des connotations est l'une des principales motivations de l'emprunt linguistique".

Peut-on considérer l'emprunt comme un procédé de traduction ou non? Faute d'équivalent (dénotatif et / ou connotatif) dans la langue cible, le traducteur introduit dans le texte de la langue d'arrivée une lexie qui n'appartient pas à cette langue. II crée ainsi un effet. restitue une atmosphère, mais se fait-il clairement comprendre du lecteur potentiel ? L'emprunt est souvent, au moins au départ, inévitable, et correspond à l'introduction d'un objet, d'une technique ou d'une pratique sociale. Il a parfois aussi une motivation sociolinguistique, voire ethnologique. Par ailleurs, de tels emprunts font parfois l'objet de notes de bas de page.

Toujours dans le cadre de la traduction d'une langue à une autre, s'accompagnant du passage d'une culture à une autre, très différente de la première, le calque sémantique et syntaxique qui consiste à introduire dans la langue cible l'emploi qui est fait d'une 
expression (lexie primaire, complexe ou composée) est une possibilité qui s'offre au traducteur. Contrairement aux emprunts, les calques sont bien intégrés au texte cible. mais ils ne sont pas nécessairement faciles à décoder pour le lecteur potentiel, en raison précisément des connotations de certains mots. qui varient d'une culture à l'autre. Cependant, même si le traducteur a recours au calque, et en raison des différences entre les langues et les cultures, aucune traduction ne peut être une copie : il s'agit toujours d'une interprétation. Par ailleurs, selon Ladmiral (1979: 104), la traduction est une condition pour la survie des langues qu elle aide à vivre. Au-delà de la langue, la traduction. aussi imparfaite soit-elle, participe à la promulgation des différentes cultures.

Avant d'examiner la traduction de quelques proverbes proposée par des auteurs ouest-africains contemporains, nous conclurons celte approche théorique par une remarque, certes banale, mais qui, toutefois, nous paraît nécessaire.

Comprendre signifie traduire, et traduire implique qu'il y ait accès préalable au sens. Selon Jakobson (1963: 97). l'opération linguistique de traduction est comparable à l'opération qui consiste à recréer du sens à partir de l'acte langagier (parole) d'autrui. D'après la conception saussurienne, le langage humain est un procès constant de traduction : la langue. une machine à traduire qui permet au sujet parlant d'exprimer par la parole ses pensées, désirs. impressions, son appartenance à une culture collective, et au récepteur de les extraire.

L'Afrique traditionnelle qui se présente comme tout à fait différente du monde de l'homme blanc qu'elle exclut est très présente dans le roman ouest-africain contemporain écrit en langue française ou anglaise. et où abondent des proverbes représentatifs de cultures collectives indigènes. Ils sont une lentative de représentation de l'univers africain et viennent enrichir les thèmes abordés. Parallèlement aux différentes variétés de langues en français et en anglais (niveaux de langue, pidgins et créoles) et aux emprunts aux langues africaines. les proverhes traduits visent à signaler l'identité distincte d'individus (personnages-types des romans), ou de groupes ethniques et à la faire connaître au lecteur venu d'ailleurs.

L'écrivain africain est confronté à un choix difficile : traduire le proverbe de la langue source de façon littérale, en lui donnant, à des degrés variables, au niveau stylistique, l'apparence d'un proverbe pour le lecteur du texte traduit; ou chercher un équivalent sémantique dans la langue cible, ce qui n'est que rarement réalisable étant donné les écarts culturels entre les deux communautés sociolinguistiques. Doit-on regretter que les auteurs africains choisissent le plus souvent de nous livrer une traduction littérale des proverbes qui nous permet toutefois de deviner la acouleur" qu'ils ont dans la langue originale, mais uniquement de la deviner? Soucieux de faire connaître leur culture et de souligner les différences avec l'Europe, la grande majorité des "auteurs-traducteurs" africains renoncent à recréer dans la langue cible les effets rhétoriques qui reposent sur des procédés formels (rime, allitération, répétition...) dans la traduction littérale qu'ils proposent des proverbes. Ceux-ci sont traduits de manière à préserver les images et lat façon de penser et de "découper» la réalité extralinguistique dans la culture et la langue d'origine, la perte stylistique entraînant néanmoins une perte des effets crés et, de ce fait. une perte sémantique.

Il nous semble que le but des auteurs africains, lorsqu ils traduisent des proverbes en anglais ou en français. est «de produire dans la langue d'arrivée l'équivalent naturel le plus proche de la langue de départ, d'abord quant à la signification puis quant au style» (Nida 1963: 19).

La traduction des proverbes est un facteur dynamique d'enrichissement culturel. Cependant. le lecteur africain et le lecteur non africain ne peuvent faire la même lecture de proverbes traduits. Pour le lecteur africain. les proverbes ont une réalité et, dans la 
majorité des cas, le renvoient à une réalité socioculturelle, y compris quand il ne connaît pas la langue originale dans laquelle le proverbe existe. (Nous admettrons qu'il y a une grande affinité entre les cultures africaines.) Dans le cas où le lecteur africain appartient à la même communauté linguistique que l' "auteur-traducteur», il est à même d'apprécier la traduction et il lui est souvent possible d'identifier le proverbe original grâce aux mots et images employés dans le proverbe traduit qui est alors un calque de l'original. En vertu des universaux culturels fondamentaux et du fait que les proverbes énoncent des vérités "générales", le lecteur non africain pourra, dans de nombreux cas, décoder le sens ou une partie du sens du proverbe traduit, sans pour autant être à même d'apprécier la traduction.

La métonymie en tant que déplacement de sens par contiguité : partie pour le tout (synecdoque) ou partie pour une autre, est fréquente dans les proverbes traduits où sont reprises les métonymies de la langue d'origine.

"When the mouth begins to laugh, it is no longer able to whistle» (Munonye, J.: Obi, H.E.B., 1969, p. 127).

Il existe dans ce proverbe une double métonymie : entre la bouche et l'individu (synecdoque) d'une part. le fait de rire et de siffler et toute activité humaine d'autre part. Il dénonce l'impossibilité de faire plusieurs choses en même temps.

- "Mais la bouche qui a tété n'oublie pas la saveur du lait" (Oyono, F. : Le vieux nègre et la médaille. Paris, Julliard, 1956, p. 17).

Ce proverbe, qui fait allusion à la reconnaissance que l'enfant garde pour sa mère, contient une métonymie entre la bouche et l'enfant d'une part, entre le lait de la mère et celle-ci d'autre part.

- "Les lèvres se ferment, de peur que la bouche ne dise tout (Oyono, F. : Le vieux nègre et la médaille. Paris, Julliard, 1956, p. 227).

Lèvres et bouche renvoient par métonymie à la personne. Ce proverbe rappelle le bienfondé de la discrétion.

aIf the tongue and the mouth quarrel, they invariably make it up because they have 10 stay in the same head" (Emecheta, B.: The Joys of Motherhood, H.E.B., 1979, p. 63).

Ici, la langue et la bouche sont associées par métonymie à deux amis proches tandis que la tête, dans un processus métaphorique, renvoie au voisinage (langue et bouche font partie de la tête, deux amis proches évoluent dans le même voisinage).

Les proverbes, dont nous avons donné quelques exemples, faisant mention des organes de la parole (lèvres, bouche, langue...) et les associant par métonymie à la personne, sont nombreux, ce qui nous paraît da à l'importance de l'oralité dans les cultures africaines.

- "As the elders said. if one finger brought oil it soiled the others" (Achebe, C.: Things Fall Apart, H.E.B., 1958, p. 87).

Le doigt est associé à la personne dans ce proverbe qui fait également allusion à un aspect «civilisationniste», le fait de «manger avec la main».

- "S'il avait le ventre derrière lui, le ventre le meltrait dans un trou" (Diop. B. : Les nouveaux contes d'Amadou Koumba. Paris. Présence africaine. 1961, p. 26).

Le ventre, par un déplacement métonymique de sens, est associé à la gourmandise.

- «Le pied qui ne reste pas sur place finit par marcher sur un étron» (Diop. B. : Contes el lavanes, Paris. Présence africaine, 1963, p. 14). 
Dans une synecdoque, le pied renvoie à la personne.

- "Quand l'homme dil à son caractère : "attends-moi ici", à peine a-1-il le dos tourné yue le caractère marche sur ses talons" (Diop. B. : Les nowie'aux comtes d"Amaden Koumba. Paris. Présence africainc, 1961, p. 411.

L'homme s'adresse à lui-même en passant par une partie de lui-même : son caractère. II s'agit d'un déplacement métonymique de sens: une partie (le caractère) pour le tout (l'homme).

- "Secouer la tête doucement ou énergiquement n'a jamais débarrassé personne de ses oreilles» (Diop. B. : Comtes et lavanes, Paris. Presence africaine, 1963. p. 29).

Ce proverbe a le même sens que le précédent : "chassez le naturel, il revient au galop". Les métonymies : tête -> personne, oreille -> caractère. y sont plus concrètes et figuratives que dans le précédent. On admet que la synecdoque est un type particulier de métonymie.

Le déplacement de sens par similarité ou comparaison implicite entre l'homme et l'animal (métaphore zoomorphique) est un procédé courant dans les proverbes traduits. La symbolique de la langue d'origine est transposée de manière directe dans la langue de la traduction. Comme pour les métonymies transcodées directement, des problèmes d'accès de sens se posent. Cependant, le recours à des métaphores différentes d'une langue à l'autre ne satisferait pas les auteurs-traducteurs du fait qu'il occulterait la culture de départ.

- "Let the kite perch and let the eagle perch too. If one says no to the other, let his wing break» (Achete, C.: Thing.s Fall Apart, H.E.B., 1958, p. 14).

Les hommes compétitifs et rivaux sont implicitement comparés au milan et à l’aigle.

"Let the hawk perch and let the eagle perch" (Achebe, C.: Dead Men's Path. H.E.B., 1972.p. 74).

Ce proverbe, qui établit une comparaison entre l'homme, le faucon et l'aigle, a le même sens que le précédent.

- aThen he quoted in lbo the words of encouragement which the bedbug was said to have spoken to her children when hot water was poured on them all. She told them not to lose heart because whatever was hot must in the end turn cold" (Achebe, $C,:$ No Longer at Ease, H.E.B., 1960), p. 144).

La mère est comparée dans une métaphore zoomorphique à une punaise et recommande patience, résignation et acceptation.

a "The mother bug told her young that hot water doesn'1 remain hot for too long; it turns cold after a while" (Emecheta. B.: The Bride Price, H.E.B., 1976, p. 142).

Ce proverbe a lè même sens que le précédent.

- "A hungry dog doxes not play with one with a full stomach" (Emecheta. B.: Second-class Cirizen. H.E.B.. 1983. p. 59).

L'homme et le chien sont comparés dans ce proverbe, qui insiste sur le fait qu'il faut être prudent dans les associations que l'on fait, ne pas fréquenter plus fortuné que soi et savoir se cantonner à sa classe sociale.

aOh, dogs say that those who have buttocks don't know how to sit on it" (Nwapa. F.: Idu. H.F.B.. 1970. p. 22). 
Ce proverbe, qui établit une comparaison entre l'homme et le chien, dénonce l'insatisfaction du genre humain qui ignore son bonheur.

- «Le lion aurait-il attendu le départ du berger pour venir dévorer la brebis ?» (Oyono, F. Une vie de boy, Paris, Julliard, 1956, p. 95).

Le lion représente les lâches, la brebis les démunis, et le proverbe dénonce la lâcheté.

- «n lion même mort effraie encore plus qu’une brebis» (Dadie, B. : Climbié, Paris, Presses Pocket, 1956, p. 151)

Les apparences sont trompeuses.

«The snake that bites stone must lose its fangs» (Munonye, J.: Obi, H.E.B., 1983, p. 115).

L'homme qui surestime ses possibilités y perd tout.

- "Be as wise as a serpent and as harmless as a dove" (Emecheta. B.: Second-class Cirizen, H.E.B., 1983, p. 26).

L'élément métaphorique est ici développé en une comparaison explicite. Le serpent est souvent symbole de la sagesse dans les cultures africaines.

- "He told the proverb of the house rat who went swimming with his friend the lizard and died from cold, for while the lizard's scale kept him dry the rat's hairy body remained wet" (Achebe, C.: No Longer at Ease, H.E.B., 1960, p. 5).

Le rat symbolise ceux qui n'ont pas de caractère, les «moutons de Panurge».

"Quand la poule suit ceux qui vont ramasser du bois mort, c'est qu'elle n'a pas vu celles qui pilent le grain" (Diop, B. : Contes et lavanes. Paris, Présence africaine, 1963, p. 99).

La poule représente ceux qui savent suivre ce que leur intérêt leur dicte.

口 «L’âne est mort! Mort, les pets sont finis» (Ousmane, S. : Véhi Ciosane, Paris. Présence africaine, 1966, p. 60).

L'âne symbolise l'homme en une métaphore zoomorphique à laquelle s'ajoute une métonymie. la cause étant associée à l'effet.

Nombreuses sont les métaphores zoomorphiques qui portent sur le thème de l'éducation, de la maternité ou de la paternité.

- "As our people say, when mother-cow is chewing grass, its young ones watch its mouth" (Achebe, C.: Things Fall Apart. H.E.B., 1958, p. 49).

aDo you know the proverb which says that when mother-cow chews giant grass her little calves watch her mouth?» (Achebe, C.: Vengeful Creditor, H.E.B.. 1972, p. 64),

a "There is a saying that every mother thinks her child is a leopard for strenght" (Amadi. E.: The Concubine, H.E.B., 1966, p. 22).

" "ll is in spite of the snake that the bush rat nurtures its youngs to maturity" (Aluko, T. M.: Kinsman and Foreman, H.E.B., 1966, p. 6).

Le rat des champs symbolise les parents qui, en dépit des difficultés représentées par le serpent. aident leurs petits à devenir adultes.

- "The son of a gorilla must dance like the father gorilla" (Nwapa, F.: Erufu, H.E.B., 1966, p. 51 ).

- "L'eau ne cuira jamais le poisson qu'elle a vu naître et qu'elle a élevé» (Diop, B. : Les nouveaux contes d'Amadou Koumba, Paris. Presence africaine, 1961. page de couverture). 
Relevons ici deux métaphores: entre la mère et l'eau, et une métaphore zoomorphique entre l'enfant et le poisson.

Les proverbes ici cités et dans lesquels ont été relevés des déplacements métaphoriques ou métonymiques de sens mettent en relief la différence réelle de l'environnement. D'autres révèlent des différences socioculturelles plus profondes :

- "S'il n'est que de vous nourrir, une seule femme suffit" (Diop, B. : Les noweanx contes d'Amadou Koumba, Paris, Présence africaine, 1961, p. 66).

- "However good a suitor might be, he was never given a bride for nothing" (Nwapa. F.: Erufu. H.E.B., 1966, p. 191).

- «Comme le disaient nos pères, qu'est-ce que la femme? Un épi de maîs, la broute quiconque a de bonnes dents" (Beti. M. : Le rom miraculé. Paris, Buchet, Chastel. 1957. p. 146).

- "Tout ce que dit le petit Maure, il l'a appris sous la tente» (Diop. B. : Les nouveaux contes d'Amadou Koumba, Paris, Présence africaine, 1961, p. 83).

-When a woman is virtuous. it is easy for her to concieve" (Emecheta, B.: The Joys of Morherhood. H.E.B.. 1979, p. 31 ).

- "Avant de soigner, il faut guérir" (Diop, B. : Les noweaux contes d'Amadou Koumba. Paris, Présence africaine, 1961,p. 86).

Dans le cas où le décodage passe par la connaissance préalable de pratiques socioculturelles précises ayant une incidence sur le mode de pensée et la perception de la réalité. les proverbes traduits semblent contenir moins de déplacements métasémiques.

En conclusion, «s'il est l'huile de palme qui fait passer les mots avec les idées", le proverbe est souvent symbolique, en fonction d'un certain contexte socioculturel. II reflète le système culturel et l'organisation sociale d'une communauté et. grâce à la fixité de sa formule. à la récurrence de métaphores et métonymies lexicalisées dans la langue originale, "transcodées» dans la langue de la traduction, il contribue à recréer une atmosphère et une façon de penser. En ce sens, la traduction littérale des proverbes semble offrir plus d'avantages qu'elle n'a d'inconvénients : en soulignant les différences culturelles entre la communauté originale et la communauté à l'intention de laquelle les proverbes sont traduits, elle respecte l'identité culturelle des deux communautés. Le traducteur est un médiateur de communication et il convient qu'il permette au lecteur de s'approcher au maximum du contexte culturel original des proverhes.

La communication unilingue est facilitée par le fait que les deux pôles de l'échange communicatif ont en commun certaines pratiques sociales : la communication plurilingue et pluriculturelle, si elle est plus difficile, reste possible grâce à la médiation du traducteur. Or, les langues ne sont pas des calques universels d'une réalité universelle. mais chacune d'elles correspond à une organisation particulière des données de l'expérience. La traduction littérale des proverbes cités a l'avantage de s'approcher le plus près possible, dans la langue cible, du "découpage" de la réalité pratiqué dans la langue source. Cependant. la tâche du traducteur demeure difficile, la transmission et la traduction d'un message s’accompagnant nécessairement d'une dissipation de son sens.

Cassirer. Sapir and latterly B. L. Whort have stressed that deep differences of language carry with them ultimate differences in the way one thinks, or looks upon the world. I should prefer not to put the matter in such a way as to suggest that certain philosophical propositions are affirmed in the one culture and denied in the other. What is really involved is the difficulty or indelerminacy of correlation'. (Brower 1959: 171-172) 


\section{Notes}

1. «Cassirer, Sapir et récemment B. L. Whorf ont montré que les différences profondes entre les langues proprement dites s'accompagnent de différences plus importantes encore quant à la laçon de penser, de considerer le monde. Je preférerais éviter de m'exprimer de telle sorte que je suggérerais que certaines propositions philosophiques sont presentes dans une culture et niées dans une autre. Cé qui est véritablement en cause, c'est la difficulté et l'imprécision de la corrélation entre les deux cultures.."

\section{REFERENCES}

BROWER, R. A. (Ed.) (1959) : On Translation, Harvard University Press.

CHUQUET. H. et M. PAILLARD (1989): Approche linguistique des problèmes de raducrion. Ophrys, Édition révisée.

DUBOIS. J. (1973) : Dictironnaire de linguisrique, Larousse.

FEDOROV. A. (1953): Introduction d la thérie de la traduction. Moscou.

(iREIMAS. A. J. (1970) : Du sens, essai sémiofique. Le Seuil, Paris.

JAKOBSON, R. (196.3) : Esasai de linguisrique générale, Paris, Minuis.

JAKOBSON. R. (1977) : Hut que'stons de poérique. Paris, Le Seuil.

L.ADMIRAL. J. R. (1979) : Traduire : theorèmes pour la traduction. Paris, Payot.

MARTIN, J. (1982) : "Essai de redétinition du concept de traduction», Babel, vol. XXVIII, $\mathrm{n}^{\circ} 2$.

MOUNIN, G. (1976) : Linguisrique et traduction, Bruxelles.

NIDA. E. : Principle's of Translation. cite par Mounin. G. (1963) : Les protiemes theoriques de la traduction. Gallimard. p. 278.

QUINE. W. V. (1959) : Meaning and Translation», R. A. Brower (Ed.). On Trunslation. Harvard University Press.

STEINFE, G. ( (1978) : «Après Babel une prétique du dire el de la traduction, Paris. Albin Michel.

VINAY, J. P. el DARBEI NET, J. (1977) : Stylistique comparée du françuis el de l'anglais. Paris, Didier. 